\title{
Mise au point d'un dispositif d'étude des effets du rayonnement sur la persistance des micro-organismes pathogènes
}

\author{
M Rougier 1*, J Fargues ${ }^{1}$, R Goujet 2 , B Itier 2, S Benateau ${ }^{3}$ \\ 1 INRA, unité de recherches en lutte biologique, campus de Baillarguet, F34982 Montferrier-sur-Lez cedex; \\ 2 INRA, unité de recherche en bioclimatologie, F78850 Thiverval-Grignon; \\ ${ }^{3}$ Strader Technique, ZA du Tertre, BP 9, F49112 Pellouailles-les-Vignes, France
}

(Reçu le 30 septembre 1994; accepté le 2 février 1995)

\begin{abstract}
Résumé - Un système destiné à étudier les "effets photiques" du rayonnement en conditions artificielles sur la persistance des micro-organismes a été mis au point. Ce système est composé de plusieurs enceintes équipées de sources radiatives (lampes à halogénures métalliques). Des filtres optiques permettent de travailler dans des gammes de longueur d'onde déterminées. Le dispositif réalisé permet de maintenir constante la température de l'échantillon durant l'irradiation quel que soit le niveau d'éclairement reçu grâce à une plaque thermorégulable. Le système est équipé d'un ensemble de mesures des facteurs climatiques : température du support d'inoculum, humidité relative de l'air, rayonnement total et rayonnement ultraviolet. Les performances du banc d'irradiation ont été étudiées. Une cartographie des éclairements énergétiques en UVA et en UVB a été établie, ainsi qu'une cartographie thermique du plan d'irradiation. Le gradient des niveaux d'éclairement en UV sur le plan d'exposition des échantillons $\left(3600 \mathrm{~cm}^{2}\right)$ dépend de la distance à la source radiative. Il se situe dans une gamme de dispersion de l'ordre de $10 \%$ à $1,50 \mathrm{~m}$ et de $20 \%$ à $0,50 \mathrm{~m}$. Le gradient thermique n'excède pas $5 \%$ dans l'ensemble des situations éprouvées. Par ailleurs, la température du support des échantillons ne s'écarte pas de la consigne de plus de $1^{\circ} \mathrm{C}$. L'humidité relative, malgré l'absence de régulation, reste stable pendant la durée d'une irradiation. Le dispositif réalisé a été adapté pour des irradiations en conditions semi-contrôlées (support d'inoculum à température constante, rayonnement solaire).
\end{abstract}

rayonnement / température / micro-organismes / dispositif d'irradiation / persistance

Summary - A device for studying the effects of radiation on the persistence of pathogenic microorganisms. $A$ system was devised for separate analysis of the photic effect and the thermal effect of solar radiation on the persistence of pathogenic microorganisms under both artificial and natural light. The objective of the authors was also to investigate these effects in varying waveband and irradiance. The irradiation chambers consisted of controlled-environment incubators with 2 high-pressure metallic halogen lamps (400 W) irradiating a large temperature-controlled platform $\left(3600 \mathrm{~cm}^{2}\right)$. The light quality was modified by using 2 long pass glass filters. In using a $295 \mathrm{~nm}$ cutting filter, the UVB irradiance ranged from 1.6 to $0.4 \mathrm{~W} \cdot \mathrm{m}^{-2}$ according to the distance from the source to the target plane. At $1.50 \mathrm{~m}$ distance the gradient of distribution of UVB irradiance on the target surface was less than $12 \%$. The thermal gradient did not exceed $5 \%$. The difference between the target temperature and the measured temperature at the sample surface support was less than $1^{\circ} \mathrm{C}$. The relative humidity remained stable around $60 \%$ during the inoculum exposure. The device has been adapted for studies in 'semi-field' conditions (constant temperature for the support of inoculum and solar radiation).

radiation / temperature / microorganisms / irradiation device / persistence

* Correspondance et tirés à part 


\section{INTRODUCTION}

Les épidémiologistes s'accordent pour reconnaître aux facteurs climatiques un rôle déterminant dans la régulation des maladies des plantes (Van der Plank, 1975 ; Schrödter, 1987 ; Rapilly, 1991) et des animaux pœcilothermes tels que les invertébrés nuisibles (Fuxa et Tanada, 1987 ; Ferron et al, 1991). En raison de la démarche globale et empirique adoptée pour leur élaboration, beaucoup de modèles opérationnels de prévision des pullulations et des épidémies, proposés au cours de ces dernières années, présentent un domaine d'application géographiquement restreint et un intérêt cognitif limité (Got, 1985 ; Brown, 1987). L'approche systémique, prenant en compte, séquence par séquence, les différentes étapes de la maladie, permet de hiérarchiser et de quantifier les effets des paramètres climatiques et in fine d'améliorer notre compréhension du fonctionnement des pathosystèmes (Rapilly, 1991).

En ce qui concerne l'épizootiologie des maladies d'insectes et l'utilisation de micro-organismes entomopathogènes en lutte microbiologique (Burges, 1981), la persistance du germe dans l'environnement constitue une étape essentielle pour la régulation des populations-hôtes visées (Tanada et Fuxa, 1987). De nombreux modèles empiriques ont été proposés pour estimer la survie des propagules entomopathogènes ou la rémanence des biopréparations insecticides à base de spores et toxine protéique cristallisée de Bacillus thuringiensis Berliner, de polyèdres de baculovirus ou de spores de champignons filamenteux (Pinnock et al, 1971, 1974, 1975 ; Brand et al, 1975, 1976 ; Burges et al, 1975 ; Beegle et al, 1981 ; Richards et Payne, 1982). Néanmoins, sauf quelques rares exceptions (Leong et al, 1980 ; Fargues et al, 1988), ces modèles n'intègrent pas les facteurs climatiques déterminant la dynamique des populations microbiennes tels que la composante ultraviolette du rayonnement solaire.

En fait, la nécessité d'améliorer la stratégie d'application des produits microbiens insecticides en fonction du climat des écosystèmes visés et de sélectionner des micro-organismes mieux adaptés à des conditions environnementales définies (Fargues et al, 1989) a amené divers auteurs à développer une méthodologie appropriée. Des dispositifs spécifiques ont été mis au point afin d'analyser les effets des facteurs climatiques, en variables séparées ou en interaction, sur la survie des propagules infectieuses. Le rayonnement solaire étant généralement considéré comme le facteur clef de la persistance des micro-organismes entomopathogènes dans le couvert végétal (Ignoffo et Hostetter, 1977; Burges, 1981 ; Benz, 1987), la plupart des travaux ont été consacrés à l'analyse de ses effets en conditions expérimentales (Burges et al, 1975 ; Ignoffo et al, 1977 ; Krieg et al, 1980 ; Zimmermann, 1982 ; Carruthers et al, 1988). Toutefois, dans ces expériences d'irradiation, 2 paramètres ont souvent été négligés : d'une part, la qualité de la bande spectrale à laquelle était soumis l'inoculum (forte proportion d'UV lointain dans Ignoffo et al, 1977) et, d'autre part, l'échauffement du support de l'inoculum irradié en raison de la proximité des sources radiatives (Zimmermann, 1982). La prise en compte de ces 2 paramètres s'étant avérée indispensable à la suite des études de persistance des conidiospores de l'hyphomycète entomopathogène Nomuraea rileyi (F) Samson in situ dans des microparcelles recouvertes d'écrans sélectifs (Fargues et al, 1988), nous nous sommes attachés à mettre au point un banc d'irradiation en vue d'analyser l'influence du rayonnement sur la persistance des micro-organismes en contrôlant la quantité d'éclairement reçue par l'inoculum irradié ainsi que sa température. Le présent article a pour objet la description et l'analyse des principales caractéristiques physiques du dispositif issu de cette recherche méthodologique, les résultats des essais microbiologiques étant présentés par ailleurs (Fargues et al, 1989, 1991 ; Rougier et al, 1992).

\section{OBJECTIFS ET MÉTHODOLOGIE}

Les dispositifs sont destinés à irradier, pendant des temps d'exposition variables, des inoculums microbiens placés sur un support (filtre en esters de cellulose ou disque de feuille maintenu en survie sur de la gélose hydratée). Après irradiation à un niveau d'éclairement déterminé pendant un temps défini, à une température contrôlée et une humidité relative connue, différents contrôles microbiologiques et/ou pathologiques permettent d'évaluer les effets du rayonnement sur les divers aspects de la persistance sensu lato d'un micro-organisme (survie des propagules, vitesse de germination des spores, activité entomopathogène ou entomotoxique) (Fargues et al, 1988, 1989, 1991) et de leur stabilité génétique (Couteaudier, Fargues et Riba, non publié). 
Les choix qui ont présidé à la conception de ce dispositif d'irradiation ont été les suivants :

- i) créer des conditions d'éclairement permettant de simuler les effets du rayonnement solaire atteignant la surface terrestre avec un spectre continu de $290 \mathrm{~nm}$ à $3000 \mathrm{~nm}$, en privilégiant le niveau d'éclairement $\left(W \cdot \mathrm{m}^{-2}\right)$ des bandes spectrales les plus actives (Fargues et al, 1988), notamment les UVB $(280-320 \mathrm{~nm})$ et les UVA $(320-400 \mathrm{~nm})$, tout en conservant les radiations de longueurs d'onde plus élevées susceptibles de modifier les réponses aux radiations ultraviolettes (Leach, 1970) ;

- ii) contrôler la température des supports d'inoculums irradiés, la proximité des sources d'éclairement artificielles provoquant un échauffement souvent sans rapport avec les niveaux de température de surface du couvert végétal in situ;

- iii) éviter la présence de radiations ultraviolettes courtes (UVC : < $280 \mathrm{~nm}$ ) par l'interposition de filtres coupe-bandes entre les sources radiatives et la chambre d'irradiation, cette disposition permettant en outre de confiner l'ozone produit par les lampes dans l'enceinte du projecteur ;

- iv) disposer de plateaux d'irradiation d'une surface suffisante pour exposer le nombre d'échan- tillons indispensable à l'analyse fine des conditions de survie des inoculums et à l'élaboration de modèles mathématiques (Smits et al, 1992) ou à l'analyse comparée de la variabilité intraspécifique de la résistance au rayonnement en vue de la sélection de micro-organismes plus tolérants (Fargues et Rougier, non publié).

\section{DESCRIPTION DU SYSTÈME RÉALISÉ}

Le dispositif est constitué de 2 enceintes d'irradiation comprenant chacune une source radiative, des filtres optiques spécifiques, une plaque support d'échantillons thermorégulable et un système de mesure des paramètres physiques pertinents (rayonnement, température de surface, humidité relative) (fig 1).

\section{Source radiative}

Deux lampes à halogénures métalliques sont mises en place au dessus d'une ouverture pratiquée à la face supérieure de chaque enceinte. Ces lampes à décharge du type HQI-TS $400 \mathrm{~W}$ (OSRAM, zone industrielle, F67120 Molsheim) sont placées dans des réflecteurs en aluminium martelé Radior 2 (OSRAM).

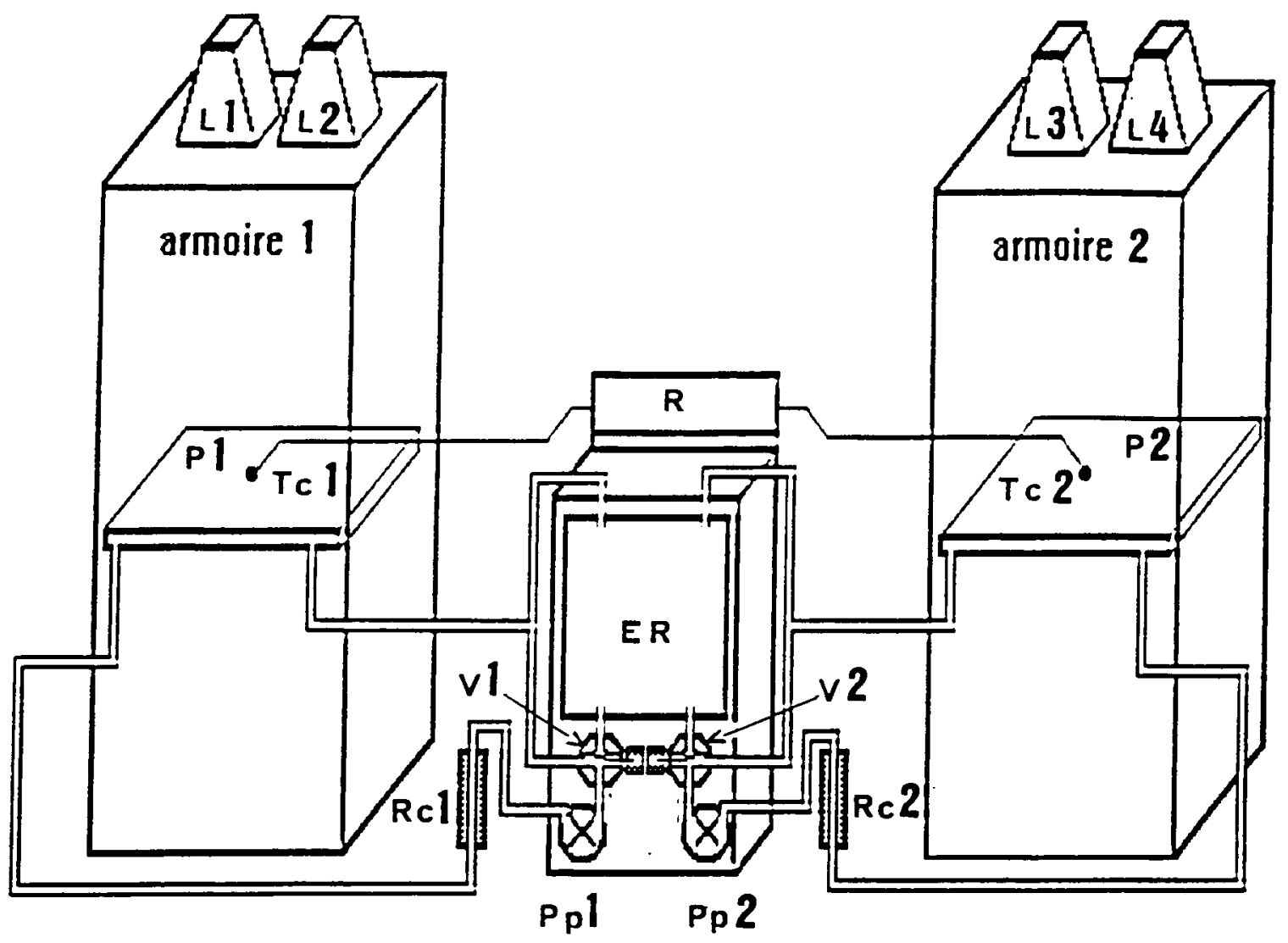

Fig 1. Schéma du dispositif d'irradiation : enceintes (armoires 1 et 2 ) ; lampes à halogénures métalliques ( $L 1$ à $L 4)$; système de thermorégulation des supports d'inoculum : thermocouples de contrôle (Tc1 et Tc2), régulation électronique $(R)$, bac à eau réfrigérée $(E R)$, vannes proportionnelles (V1 et $\mathrm{V} 2)$, pompes ( $\mathrm{Pp} 1$ et $\mathrm{Pp} 2)$, résistances chauffantes (Rc1 et $\mathrm{Rc} 2$ ), plaques à circulation d'eau ( $\mathrm{P} 1$ et P2). 
La vitre en verre trempé du réflecteur est remplacée par un filtre optique coupe-bande spécifique $(250 \mathrm{x}$ $220 \times 3 \mathrm{~mm}$ ) fabriqué sur commande par Schott Glaswerke (Schott France, 8, rue Fournier, F92110 Clichy). Les filtres optiques utilisés présentent un degré de transmission intrinsèque inférieur à $1 \times 10^{-5}$ dans les longueurs d'onde de l'ultraviolet $C(<280$ $\mathrm{nm}$ ). Cette disposition permet de limiter la pollution en ozone dans le volume de l'enceinte en-dessous du seuil de détection (mesures effectuées au moyen du tube réactif Drager ozone 0,05 b - Drager-Brandt SA, 30 , route de la Fédération, BP 141, F67025 Strasbourg cedex).

Les variations de flux lumineux consécutives aux variations de tension du secteur ont été considérablement réduites en interposant un régulateur sinusoïdal de type Réguvolt A 73.30.50 (VP Électronique, 9, rue G-Péri, F93100 Massy).

\section{Support d'irradiation thermorégulable}

La régulation thermique du dispositif est double. La température de l'air de l'enceinte, d'un volume de $0,605 \mathrm{~m}^{3}(1,55 \times 0,63 \times 0,62 \mathrm{~m})$ est régulée avec un système conventionnel (comportant notamment un groupe froid à refroidissement par air d'une puissance frigorifique de $730 \mathrm{fg} \mathrm{h}^{-1}$ et d'une puissance électrique de $3 / 8 \mathrm{CV}$ ).

Néanmoins, la régulation de l'air ambiant n'est pas suffisante pour éviter un échauffement des supports d'inoculum au cours des irradiations. C'est pourquoi un système original de régulation de la température de surface des supports irradiés a été mis au point (fig 1). La plupart des auteurs (Leach, 1971; Maddison et Manners, 1972) considèrent que la température des micro-organismes est très proche de la température de surface du support sur lequel ils ont été déposés. Cette dernière est estimée au moyen d'un thermocouple Tc (T40-Thermoelectric ; Équipement Scientifique, 127, rue de Buzenval, F92380 Garches) ; il est abrité du rayonnement direct par une bille de verre opaque et maintenu en contact avec le filtre support, fixé par 4 morceaux d'adhésif double face sur le fond d'une boîte de Petri de $5 \mathrm{~cm}$ de diamètre. La conduction thermique entre la boite et la plaque thermorégulable est améliorée en interposant une couche d'environ $0,2 \mathrm{~mm}$ de glycérol ou de pâte thermoconductrice aux silicones.

Une température de consigne est affichée sur un système d'asservissement (R) (Strader Technique, F48112 Pellouailles-les-Vignes). La température de surface de l'échantillon mesurée par le thermocouple (Tc) est comparée à cette valeur de consigne et la régulation est assurée par circulation d'eau thermostatée à travers la plaque. Selon que la température des échantillons est supérieure ou inférieure à la température de consigne, l'eau peut soit provenir du bac réfrigéré (ER), soit circuler en boucle avec possibilité d'apport calorifique grâce à une résistance chauffante (Rc). La sélection s'effectue au moyen d'une vanne proportionnelle 3 voies (V).

\section{Système de mesure des facteurs physiques}

Une série de capteurs connectés à une acquisition de données Campbell 21 X (Campbell Scientific Ltd, College Road, Sutton Borington, Loughrough LE125RA, Royaume-Uni) permet le suivi en continu des conditions climatiques régnant dans l'enceinte. Les grandeurs mesurées sont, outre la température de surface du support d'inoculum, I'humidité relative de l'air, mesurée au moyen d'un capteur capacitif (Vaisala-Humicap type $1638 \mathrm{HM}$; Étoile International, 113, rue Cardinet, F75017 Paris), le rayonnement de 300 à $2500 \mathrm{~nm}$ grâce à un pyranomètre (CIMEL CE $180 ; 5$, cité de Phalsbourg, F75011 Paris). Les flux d'UVB (280-320 $\mathrm{nm})$ et d'UVA (320-400 nm) sont aussi mesurés ponctuellement avec un radiomètre OSRAM UV Centra.

\section{RÉSULTATS}

L'analyse des conditions auxquelles sont soumis les micro-organismes irradiés concerne essentiellement la distribution spectrale du rayonnement reçu, l'homogénéité du niveau d'éclairement sur le plan d'irradiation et la variabilité de la température des échantillons. À l'échelle de temps où sont menées les études des effets du rayonnement dans des conditions de simulation solaire, les effets de l'humidité de l'air sur la persistance des micro-organismes sont négligeables (Clerk et Madelin, 1965 ; Bouamama-Benhalima, 1992) ; cependant, les variations de I'humidité ont été aussi prises en compte.

\section{Répartition spectrale et niveau d'éclairement}

La répartition spectrale du rayonnement reçu au niveau des échantillons a été établie à l'aide d'un spectroradiomètre LICOR LI-800 (Cunow, 11, avenue de l'Ados, F95800 Cergy-Saint-Christophe).

La comparaison des flux d'UV reçus au niveau du plan d'irradiation avec les flux d'UV naturel par ciel clair et par ciel couvert montre que la source radiative utilisée présente une gamme de radiations ultraviolettes voisine de celle du rayonnement solaire (fig 2).

En faisant varier la distance entre la source radiative et le plan d'irradiation $(0,50 \mathrm{~m}$ à $1,50 \mathrm{~m}$ ), on obtient une gamme de niveaux d'éclairement qui permet d'éprouver des conditions proches des conditions naturelles (tableáu I).

Les résultats des mesures effectuées sur des plans horizontaux à 3 distances de la source de rayonnement : $50 \mathrm{~cm}, 1 \mathrm{~m}$ et $1,50 \mathrm{~m}$ en 20 points 


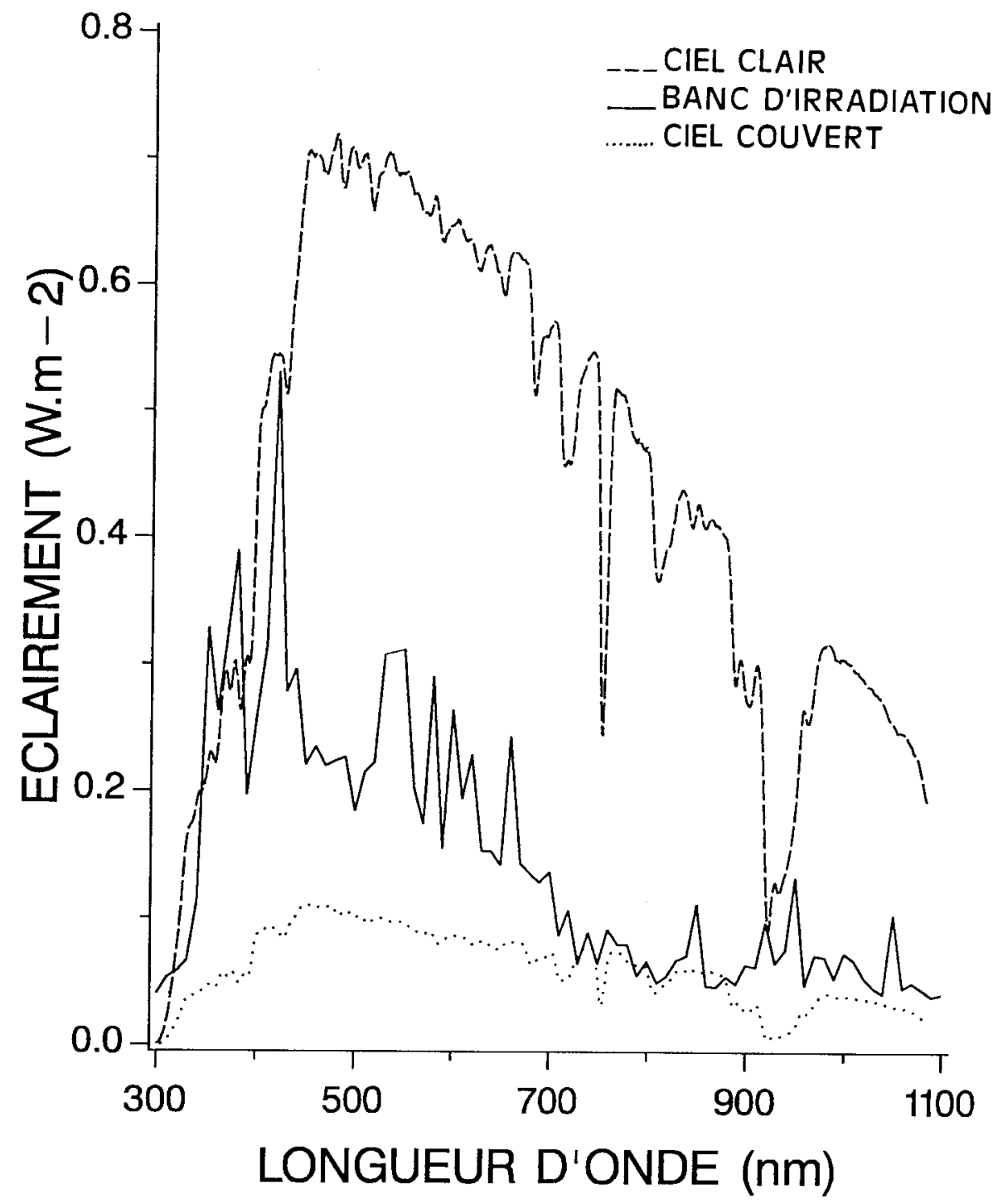

Fig 2. Comparaison des compositions spectrales du rayonnement reçu sur un plan horizontal en banc d'irradiation (à $1 \mathrm{~m}$ de la source) et du rayonnement solaire incident par ciel clair et par ciel couvert.

Tableau I. Variations de flux (W. $\left.\mathrm{m}^{-2}\right)$ dans les bandes spectrales ultraviolettes A (320-400 nm) et B (280-320 nm) mesuré en banc d'irradiation en fonction de la distance à la source et valeurs de flux solaire correspondantes mesurées par ciel clair au cours de la journée (La Minière, $48^{\circ} 51^{\prime} \mathrm{N}, 2^{\circ} 06^{\prime} \mathrm{E}, 5$ juillet 1991).

Nature du rayonnement mesuré

$$
\text { Éclairement énergétique en UVB et en UVA }\left(W . m^{-2}\right)
$$

En simulation solaire

Sans filtre optique Avec filtre WG 295

Distance à la source (m)

$\begin{array}{lrrr}\text { UVA } & 0,50 & 59,6 & 52,8 \\ \text { UVB } & & 4,7 & 1,6 \\ \text { UVA } & & & \\ \text { UVB } & 1,00 & 29,8 & 25,7 \\ & & 2,4 & 0,7 \\ \text { UVA } & & & \\ \text { UVB } & 1,50 & 16,6 & 14,6 \\ & & 1,2 & 0,4\end{array}$

\section{En conditions naturelles}

Heure de mesure Sans filtre optique (UTC)

$\begin{array}{lr}\text { entre } 11 \mathrm{~h} & 45,0 \\ \text { et } 13 \mathrm{~h} & 1,6 \\ & \\ \text { vers } 8 \mathrm{~h} \text { et } & 25,0 \\ \text { vers } 16 \mathrm{~h} & 0,7 \\ & \\ \text { vers } 7 \mathrm{~h} \text { et } & 14,0 \\ \text { vers } 17 \mathrm{~h} & 0,3\end{array}$


équidistants ont permis de quantifier les variations spatiales du flux d'ultraviolet $A$ et $B$. Ainsi, pour I'UVB à $50 \mathrm{~cm}$, les valeurs sont comprises entre 5,5 et $4,3 \mathrm{~W} \cdot \mathrm{m}^{-2}$ (dispersion de $23 \%$ ), à $1 \mathrm{~m}$ entre 2,9 et $2,5 \mathrm{~W} \cdot \mathrm{m}^{-2}$ (dispersion de $16 \%$ ) et à $1,5 \mathrm{~m}$ entre 1,7 et $1,5 \mathrm{~W} \cdot \mathrm{m}^{-2}$ (dispersion $11 \%$ ). On a représenté sur la figure 3 les courbes d'isorayonnement pour une distance de $1 \mathrm{~m}$ à la source.

En ce qui concerne I'UVA, les valeurs sont comprises entre 52 et $41 \mathrm{~W} \cdot \mathrm{m}^{-2}$ (soit une dispersion de $21,3 \%$ ) à $50 \mathrm{~cm}$ de la source; à $1 \mathrm{~m}$ ces valeurs deviennent 28,5 et $24 \mathrm{~W} . \mathrm{m}^{-2}$ (dispersion : $15,8 \%$ ) et à $1,50 \mathrm{~m}$ les flux varient entre

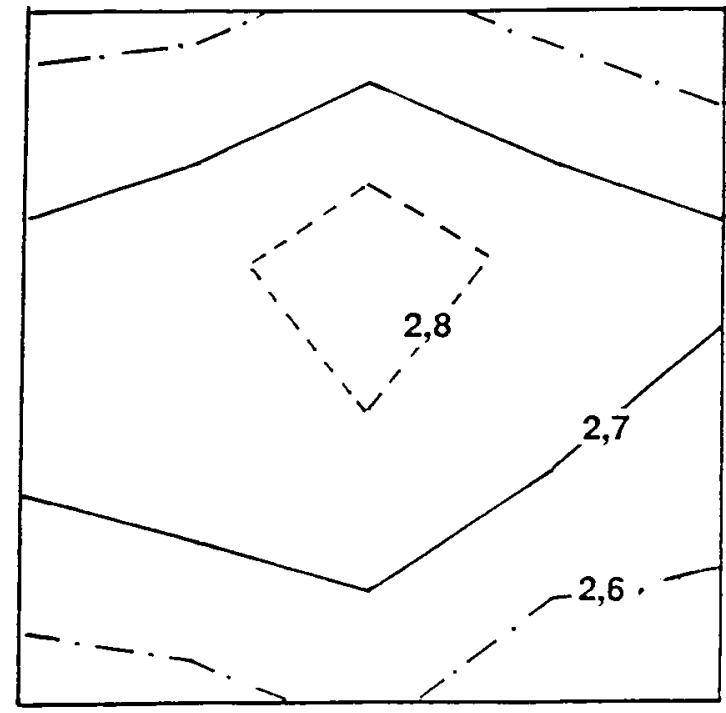

Fig 3. Courbe d'isorayonnement en UVB (W.m-2) reçu sur le plan horizontal à $1 \mathrm{~m}$ des sources (mesures effectuées en l'absence de filtre optique)

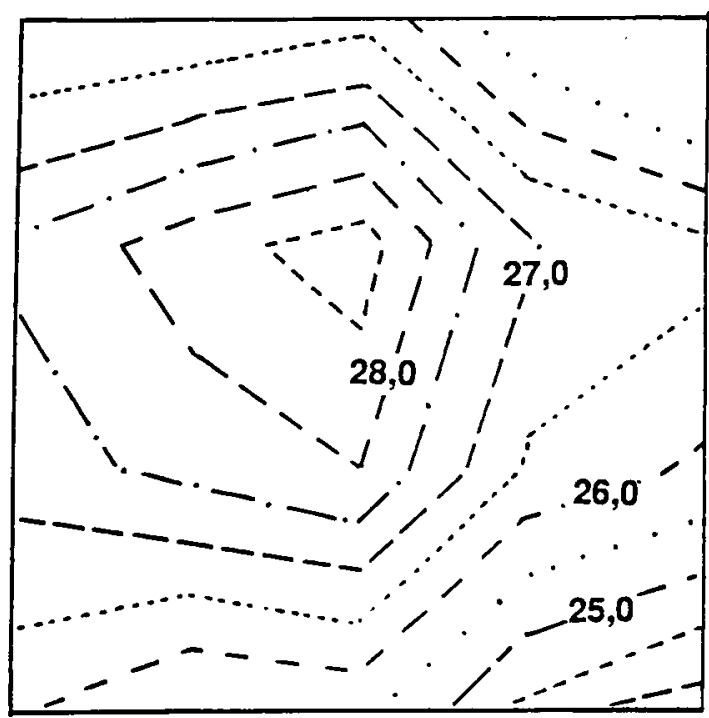

Fig 4. Courbe d'isorayonnement en UVA (W.m-2) reçu sur le plan horizontal à $1 \mathrm{~m}$ des sources (mesures effectuées en l'absence de filtre optique).
17,7 et 16 W. $\mathrm{m}^{-2}$ (dispersion de l'ordre de $10 \%$ ). La figure 4 représente les courbes d'isorayonnement en UVA pour des mesures effectuées à $1 \mathrm{~m}$ de la source.

\section{Variation de la température des échantillons irradiés}

Suivant un protocole analogue à celui adopté pour I'UV, des mesures effectuées avec 20 thermocouples ont permis de tracer les courbes d'isotempérature pour une température de consigne de $20^{\circ} \mathrm{C}$, respectivement à $0,50 \mathrm{~m}$ et $1 \mathrm{~m}$ des sources (fig 5). On constate que la température de surface varie de $\pm 1^{\circ} \mathrm{C}$ par rapport à la température de consigne.
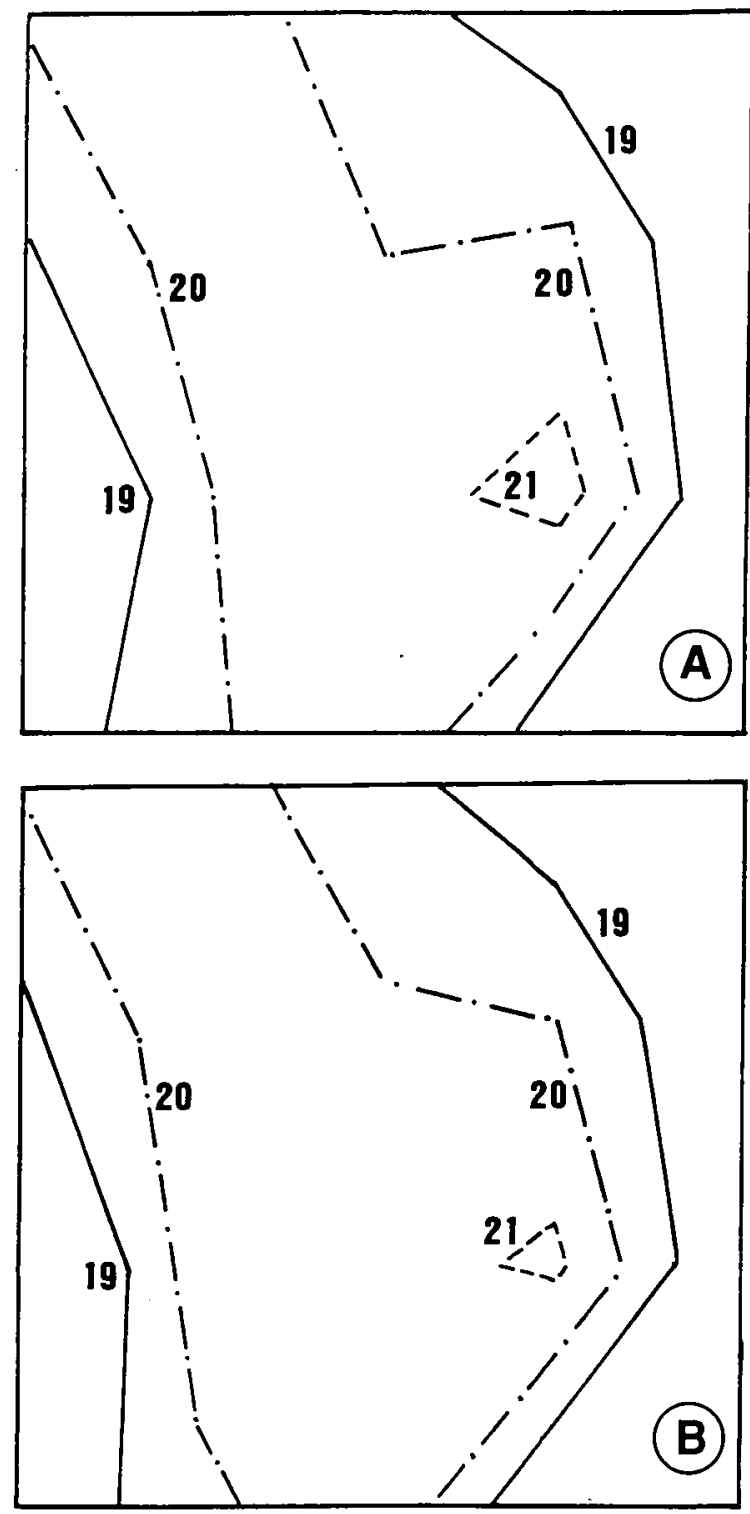

Fig 5. Courbe d'isotempérature ( $\mathrm{C}$ ) sur le plan horizontal, A à $1 \mathrm{~m}$ de la source; $\mathrm{B}$ : à $0,5 \mathrm{~m}$ de la source. 


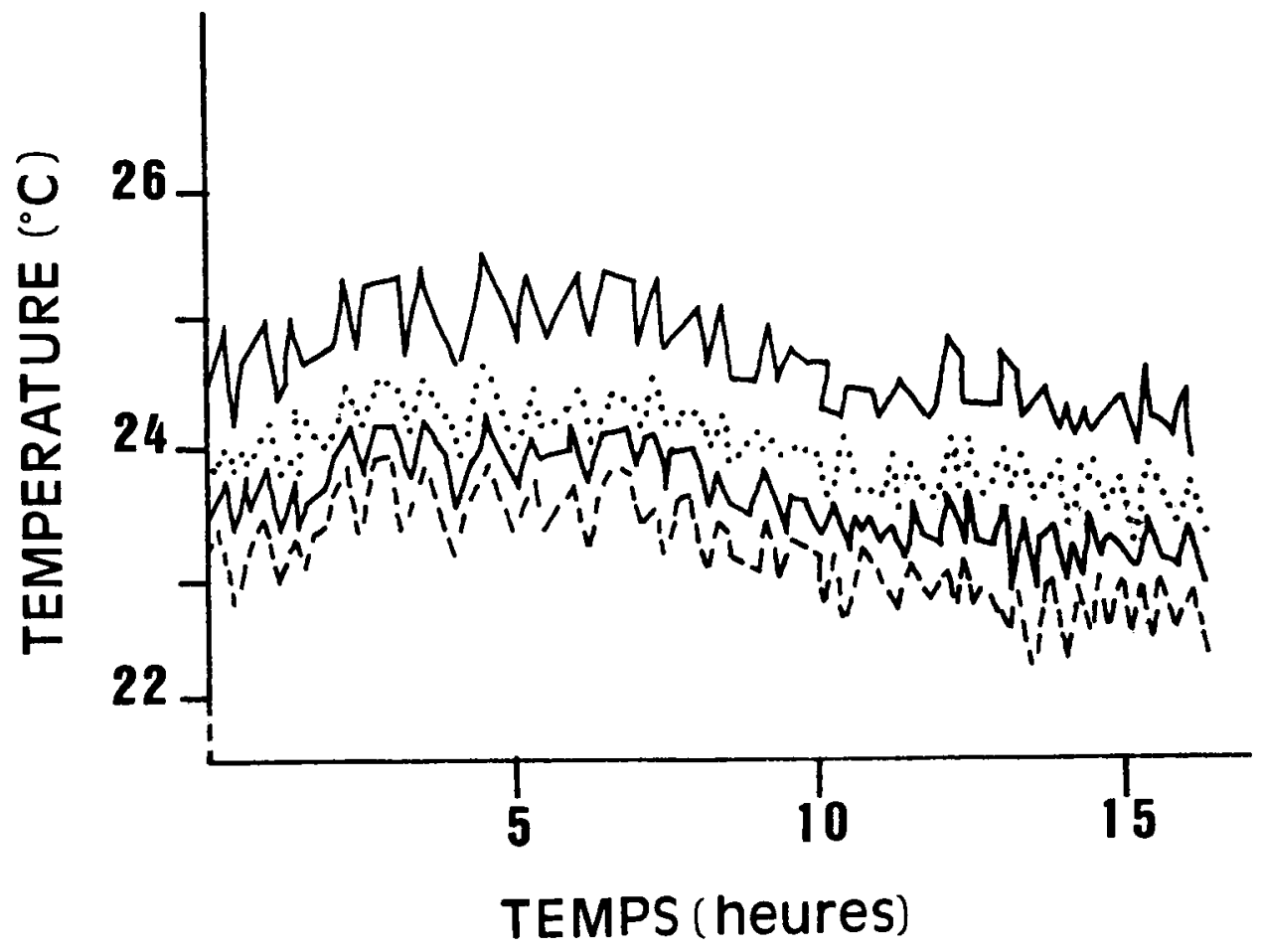

Fig 6. Variation de la température de 4 échantillons en fonction du temps.

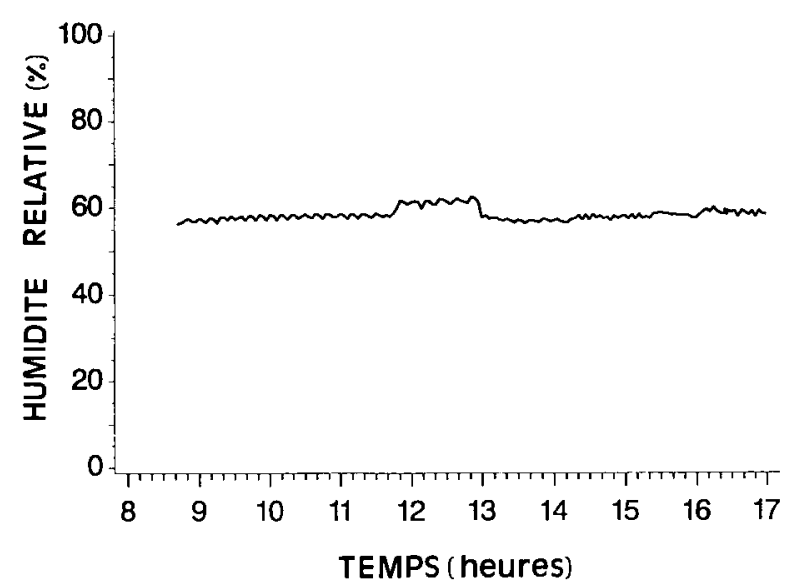

Fig 7. Variation de l'humidité relative de l'air à l'intérieur de l'enceinte d'irradiation en fonction du temps.

L'évolution de la température de surface de 4 échantillons placés sur la plaque thermorégulée a.été suivie pendant $15 \mathrm{~h}$ (fig 6 ). On constate une très bonne stabilité dans le temps en un point donné $\left( \pm 0,5^{\circ} \mathrm{C}\right)$.

\section{Variation de l'humidité de l'air dans l'enceinte}

La mise en place sur le fond de l'enceinte d'un bac $(30 \times 30 \times 10 \mathrm{~cm})$ avec de l'eau $(5 \mathrm{~cm})$ permet d'assurer la régulation de l'humidité à un niveau sensiblement constant pendant une séquence d'irradiation de l'ordre de $3 \mathrm{~h}$ (fig 7 ).

\section{Adaptation du dispositif aux irradiations solaires}

En raison de la variabilité des conditions d'ensoleillement, les relations établies en conditions artificielles doivent être confirmées par des expériences d'irradiation conduites en rayonnement solaire. Le dispositif expérimental a donc été adapté dans ce sens. La modification principale a consisté à installer les plaques thermorégulées sur des plates-formes pivotant suivant 2 axes, de manière à maintenir le plan d'irradiation à la normale par rapport aux rayons solaires incidents (fig 8).

Sur le plan technique, l'orientation et l'inclinaison des plaques sont assurées par 2 mécanismes motorisés pilotés par un système optoélectronique à photodiodes (Goujet, non publié). Pour assurer le maintien des supports d'inoculums, une plaque de chlorure de polyvinyle alvéolée a été adaptée sur chacune des plaques. Par ailleurs, pour faciliter les études de l'effet de la température sur la sensibilité au rayonnement, la régulation électronique des 2 plaques a été modifiée afin de permettre de travailler simultanément à 2 températures différentes. 


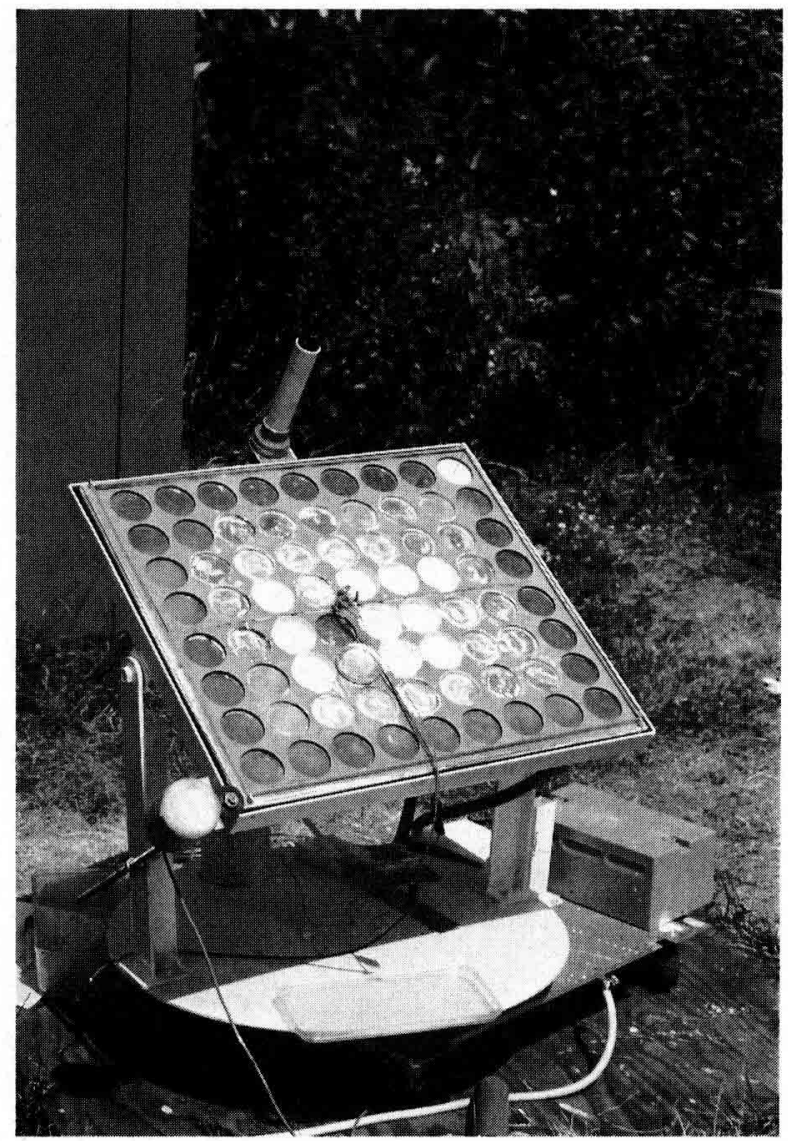

Fig 8. Plaque thermorégulée orientable et inclinable pour les irradiations en conditions naturelles (rayonnement solaire).

\section{DISCUSSION ET CONCLUSION}

L'étude des conditions d'irradiation montre que le dispositif proposé répond bien au cahier des charges qui avait présidé à sa mise au point.

II faut néanmoins souligner que l'utilisation des lampes à halogénures métalliques HQI-TS comme sources radiatives résulte d'un choix raisonné par rapport aux simulateurs solaires équipés de lampes au xénon (Carruthers et al, 1988 ; Stevenson et Pennypacker, 1988). Ces derniers sont plus performants en matière d'homogénéité de flux et de qualité du rayonnement (par rapport au spectre solaire), mais ils offrent des surfaces d'irradiation inférieures à $700 \mathrm{~cm}^{2}$ (au lieu des $3600 \mathrm{~cm}^{2}$ dans le système proposé) difficilement compatibles avec des études quantitatives nécessitant l'analyse de nombreux échantillons. Par ailleurs, la qualité de la régulation de la température des supports d'inoculum obtenue avec le dispositif décrit permet d'aborder l'analyse des interactions entre les effets photiques et les effets thermiques du rayonnement. Récemment, Smits et al (1992) ont ainsi pu montrer que des températures non létales $\left(35^{\circ} \mathrm{C}\right)$ sensibilisent les spores quiescentes de l'hyphomycète entomopathogène Paecilomyces fumosoroseus (Wize) Brown et Smith aux UV naturels (par rapport à $25^{\circ} \mathrm{C}$ ), alors que Krieg et al (1980) et Zimmermann (1982) n'avaient pas tenu compte des effets de l'accroissement de la température $\left(23,5\right.$ à $\left.36^{\circ} \mathrm{C}\right)$ sur la sensibilité de divers inoculums entomopathogènes (baculovirus de Mamestra brassicae, $B$ thuringiensis et Metarhizium anisopliae (Metsch) Sorok, aux radiations ultraviolettes. Dans certains cas, tels que les irradiations des propagules sur milieu gélosé hydraté, cet aspect a pu être négligé en raison du refroidissement de la surface du gel dû à l'évaporation de l'eau (Maddison et Manners, 1973).

Le banc d'irradiation et sa variante mobile permettant de travailler en conditions semi-contrôlées sous rayonnement solaire constituent des outils privilégiés pour l'analyse quantitative des effets du rayonnement sur la survie des microorganismes.

\section{REMERCIEMENTS}

Le banc d'irradiation en conditions contrôlées a été réalisé grâce aux crédits attribués par I'INRA dans le cadre de l'Action incitative programmée intersecteur "Climat et germes/épidémiologie» (1986-1988). La réalisation de sa variante mobile destinée aux expositions au rayonnement solaire a été financée par le Service de la recherche, des études et du traitement de l'information sur l'environnement (SRETIE) du ministère de l'Environnement (subvention $n^{\circ} 89337$; 1989-1990).

\section{RÉFÉRENCES}

Beegle CC, Dulmage HT, Wolfenbarger DA, Martinez E (1981) Persistence of Bacillus thuringiensis Berliner insecticidal activity on cotton foliage. Environ Entomol 10, 400-401

Benz G (1987) Environment. In : Epizootiology of Insect Diseases (JR Fuxa, Y Tanada, eds), John Wiley, New York, 177-214

Bouamama-Benhalima N (1992) Effet de l'eau en phase vapeur et en phase liquide sur la survie des conidiospores de Paecilomyces fumosoroseus (Wize) Brown et Smith, hyphomycète pathogène de Spodoptera frugiperda JE Smith (Lep Noctuidae) en conditions contrôlées. Thèse doc, univ Paris Vl, $86 \mathrm{p}$

Brand RJ, Pinnock DE, Jackson KL, Milstead JE (1975) Methods for assessing field persistence of 
Bacillus thuringiensis spores. J Invertebr Pathol 25, 199-208

Brand RJ, Pinnock DE, Jackson KL, Milstead JE (1976) Viable spore count as an index of effective dose of Bacillus thuringiensis. J Invertebr Pathol 27, 141-148

Brown GC (1987) Modeling. In : Epizootiology of Insect Diseases (JR Fuxa, Y Tanada, eds), John Wiley, New York, 43-68

Burges HD (1981) Microbial Control of Pests and Plant Diseases 1970-1980. Academic Press, New York, $949 \mathrm{p}$

Burges HD, Hillyer S, Chanter DO (1975) Effect of ultraviolet and gamma rays on the activity of delta endotoxin protein crystal of Bacillus thuringiensis. $J$ Invertebr Pathol 25, 5-9

Carruthers RI, Feng Z, Ramos ME, Soper RS (1988) The effect of solar radiation on the survival of Entomophaga grylli (Entomophthorales: Entomophthoraceae) conidia. J Invertebr Pathol 52, 154-162

Clerk GC, Madelin MF (1965) The longevity of conidia of 3 insect-parasitizing hyphomycetes. Trans Brit Mycol Soc 48, 193-209

Fargues J, Rougier M, Goujet R, Itier B (1988) Effet du rayonnement solaire sur la persistance des conidiospores de l'hyphomycète entomopathogène, Nomuraea rileyi, à la surface du couvert végétal. Entomophaga 33, 357-370

Fargues J, Rougier M, Goujet R, Itier B, Coustere C (1989) Microclimatic studies of the persistence of Nomuraea rileyi. IOBC/WPRS Meeting "Microbial Control of Weevils and Environmental Persistence of Pathogens and Nematodes", Versailles, IOBC/WPRS Bull 12, 64-65

Fargues J, Rougier M, Goujet R, Coustere C, Itier B (1991) Paecilomyces fumosoroseus persistence: inactivation of conidia by simulated sunlight radiations. 2nd IOBCMPRS Meeting "Microbial Control of Pests", Rome IOBC/WPRS Bull 14, 82

Ferron P, Fargues J, Riba G (1991) Fungi as microbial insecticides against pests. In : Handbook of Applied Mycology (DK Arora, L Ajello, KG Mukerji, eds), vol 2, 665-706

Fuxa JR, Tanada Y (1987) Epizootiology of insect Diseases. John Wiley and Sons, New York

Got B (1985) Approche de la modélisation de la dynamique de population de la pyrale du maïs (Ostrinia nubilalis $\mathrm{Hbn}$ ) (Lepidoptera: Pyralidae) dans le bassin parisien. Thèse doc, univ Paris VII, $210 \mathrm{p}$

Ignoffo CM, Hostetter DL (1977) Environmental stability of microbial insecticides. Misc Publ Entomol Soc Am 10, 1-80

Ignoffo CM, Hostetter DL, Sikorowski PP, Sutter G, Brooks WM (1977) Inactivation of representative species of entomopathogenic viruses, a bacterium, fungus and protozoan by an ultraviolet light source. Environ Entomol 6, 411-415

Krieg A, Gröner A, Huber J, Matter M (1980) Über die Wirkung von mittel- und langwelligen ultravioletten
Strahlen (UV-B und UV-A) auf insektenpathogene Bakterien und Viren deren Beeinflüssung durch UVSchutzstoffe. Nachrichtenbl Dtsch Pflanzenschutzd 32, 100-105

Leach DM (1970) A pratical guide to the effects of visible and ultraviolet light on fungi. Tech Paper The Oregon Agricultural Experiment Station, 2540, 610-664

Leong KLH, Camo RJ, Kubinski AM (1980) Factors affecting Bacillus thuringiensis total field persistence. Environ Entomo/ 9, 593-599

Maddison BC, Manners JG (1972) Sunlight and viability of cereal rust uredospores. Trans $\mathrm{Br} \mathrm{Mycol}$ Soc $59,429-443$

Maddison BC, Manner JG (1973) Lethal effects of artificial ultraviolet radiation on cereal rust uredospores. Trans Br Mycol Soc 60, 471-494

Pinnock DR, Brand RJ, Milstead JE (1971) The field persistence of Bacillus thuringiensis spores. $J$ Invertebr Pathol 18, 405-411

Pinnock DE, Brand RJ, Jackson KL, Milstead JE (1974) The field persistence of Bacillus thuringiensis spores. J Invertebr Pathol 23, 341-346

Pinnock DE, Brand RJ, Milstead JE, Jackson KL (1975) Effect of tree species on the coverage and field persistence of Bacillus thuringiensis spores. $J$ Invertebr Pathol 25, 209-214

Rapilly $F$ (1991) L'épidémiologie en pathologie végétale. Mycoses aériennes. INRA, Paris, $317 \mathrm{p}$

Richards MG, Payne CC (1982) Persistence of baculoviruses on leaf surfaces. Proc IIIrd Int Coll Invertebr Pathol, Brighton, 296-301

Rougier M, Fargues J, Goujet R, Smits N (1992) Photic effect of solar UV radiations on the survival of entomopathogenic hyphomycete. In : Crop structure and light microclimate: characterisation and application (C Varlet-Grancher, R Bonhomme, eds), coll Science Update, INRA, Paris, 433-438

Schrödter H (1987) Wetter und Pflanzenkrankheiten biometerologische Grundlagen der Epidemiologie. Springer Verlag, Berlin, $187 \mathrm{p}$

Smits N, Rougier M, Goujet R, Bonhomme R, Fargues $J(1992)$ Effect of solar radiation on the persistence of quiescent conidia of the Hyphomycete Paecilomyces fumosoroseus. In: Proc XXVth Annu Meeting Soc Invertebr Pathol, Heidelberg, 160

Stevenson RE, Pennypacker SP (1988) Effect of radiation, temperature and moisture on conidial germination of Alternaria solani. Phytopathology 78, 926930

Tanada Y, Fuxa JR (1987) The pathogen population. In : Epizootiology of Insect Diseases (JR Fuxa, $Y$ Tanada, eds), John Wiley and Sons, New York, 113-157

Van der Plank JR (1975) Principles of Plant Infection. Academic Press, New York, $216 p$

Zimmermann $G$ (1982) Effect of high temperatures and artificial sunlight on the viability of conidia of Metarhizium anisopliae. J Invertebr Pathol 40, 36-40 